\title{
Plattenepithel-NSCLC
}

\section{IgG1-Antikörper ermöglicht erste zielgerichtete Therapie}

Auch für Patienten mit fortgeschrittenem nicht-kleinzelligem Lungenkarzinom (NSCLC) vom Plattenepitheltyp gibt es nun eine erste zielgerichtete Therapie, sofern der Tumor den EpidermalGrowth-Factor-Rezeptor (EGFR) exprimiert, was bei $75 \%$ der Patienten der Fall ist. Seit April 2016 steht für die First-Line-Therapie der IgG1-Antikörper Necitumumab (Portrazza ${ }^{\oplus}$ ) zur Verfügung. Zusätzlich zu Gemcitabin/Cisplatin verabreicht, verlängert der Antikörper das mediane Gesamtüberleben von 10 auf 11,7 Monate.

Wie Prof. Wolfgang Schütte, Chefarzt am Krankenhaus Martha-Maria in Halle/Saale, berichtete, basiert die Zulassung auf den Ergebnissen der SQUIRE-Studie mit 1.093 Chemotherapie-naiven Patienten. Der Antikörper wurde bei maximal sechs Chemotherapiezyklen jeweils an den Tagen 1 und 8 vor Gemcitabin verabreicht. Anschließend wurde die Antikörper-Therapie allein bis zum Tumorprogress fortgeführt.

\section{Klinisch relevante Verlängerung des Überlebens}

Die Überlebensverlängerung von knapp zwei Monaten sei klinisch relevant, so Schütte. Auf das progressionsfreie Überleben hat die Therapie nur einen geringen Effekt. An Nebenwirkungen ist v.a. ein Rash zu erwarten. Patienten mit dieser Nebenwirkung scheinen besser auf den neuen Antikörper anzusprechen.

Wie Dr. Sylvia Gütz, Chefärztin der Klinik für Pneumologie und Kardiologie am Evangelischen Diakonissenkran- kenhaus in Leipzig, berichtete, ist das Plattenepithelkarzinom der Lunge mit ca. $40 \%$ bei Männern und $20 \%$ bei Frauen leicht rückläufig, aber schwerer zu behandeln als das Adenokarzinom. Die Tumoren sitzen oft zentral, die Patienten sind meist starke Raucher, bei Diagnose häufig schon älter und multimorbid.

Bei fortgeschrittener oder metastasierter Erkrankung stehen laut Gütz in der First Line Platin plus Gemcitabin/ Necitumumab, Platin plus Taxane oder Platin plus Vinorelbin zur Verfügung. In der Second Line nannte sie Docetaxel, Docetaxel plus Ramucirumab, Erlotinib und Nivolumab.

\section{Dr. Dirk Einecke}

- Launch-Pressekonferenz „Portrazza ${ }^{\oplus}$ :Zielgerichtete Therapie bei EGFR-positivem plattenepithelialen NSCLC"; Bad Homburg, April 2016 (Veranstalter:Lilly Deutschland)

\section{Awareness für herzkranke Kinder schaffen}

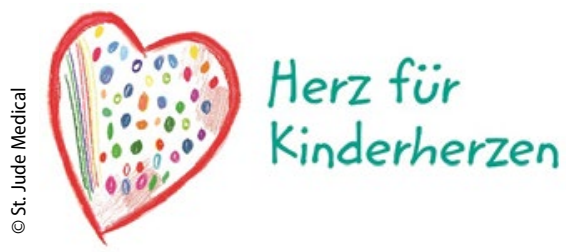

_ St. Jude Medical, ein Medizingerätehersteller, gibt auf der gemeinsamen Jahrestagung der Deutschen Gesellschaft für Thorax-, Herz- und Gefäßchirurgie und der Deutschen Gesellschaft für Pädiatrische Kardiologie den Start der Awareness-Kampagne „Herz für Kinderherzen“ bekannt. „Herz für Kinderherzen“" wurde gegründet, um Kinder mit Herzkrankheiten, ihre Eltern und Betreuer enger miteinander $\mathrm{zu}$ vernetzen und die Kommunikation zwischen den Betroffenen zu verbessern.

Angeborene Herzfehler sind bei Kindern in Deutschland die häufigste Form von Geburtsfehler. Jedes Jahr kommen hierzulande mehr als 6.500 Kinder mit einer schweren angeborenen Herzerkrankung zur Welt. Nahezu 25 \% dieser Kinder benötigen in ihrem ersten Lebensjahr mindestens einen operativen Eingriff.

Angestoßen wird die Kampagne mit einer Spende an den Bundesverband Herzkranke Kinder, die größte Patientenorganisation für Kinder mit einem angeborenen Herzfehler und ihre Eltern. Vor der Operation übergibt der Verband den Eltern der herzkranken Kinder das Kinderbuch „Annas Herzoperation“ und eine Puppe namens Erwin, der herausnehmbare Plüsch-Organe besitzt. So können die Eltern ihrem Kind die Ängste nehmen und die Situation mit ihm besprechen. Weitere Informationen unter: sjm.de/herzfuerkinderherzen. -

Red.

- Nach Informationen von St. Jude Medical

\section{Kurz notiert}

Beginn einer neuen Ära in der Behandlung von Patienten mit Krätze $\rightarrow$ Am 1. Mai 2016 hat InfectoPharm in Deutschland Scabioral ${ }^{\oplus}$ $3 \mathrm{mg}$ Tabletten mit dem verschreibungspflichtigen Wirkstoff Ivermectin eingeführt. Damit steht erstmals eine orale Behandlungsoption der Scabies zur Verfügung. Bislang musste die durch Krätzemilben hervorgerufene, stark juckende und ansteckende Hauterkrankung mittels einer topischen Ganzkörperbehandlung therapiert werden - eine Herausforderung für den Patienten, die nicht selten mit Therapieversagen im Sinne von Rezidiven verbunden ist.

Die Gabe von Tabletten stellt demgegenüber einen Innovationssprung dar, denn die Einmalgabe verbessert in erheblichem $\mathrm{Maße}$ die $\mathrm{Pa}$ tienten-Compliance. Scabioral ${ }^{\circledR} 3 \mathrm{mg}$ Tabletten sind voll erstattungsfähig und werden zur Behandlung der Scabies in einer Dosis von $200 \mu \mathrm{g}$ pro kg Körpergewicht verabreicht. Die orale Behandlungsoption eignet sich hervorragend zur synchronen Behandlung aller engen Kontaktpersonen der Patienten sowie zum Einsatz in Gemeinschaftseinrichtungen. 